\title{
Enhanced Oral Bioavailability of Curcumin via a Solid Lipid-Based Self- Emulsifying Drug Delivery System Using a Spray-Drying Technique
}

\author{
Yi-Dong Yan, ${ }^{a}$ Jung Ae KIm, ${ }^{a}$ Mi Kyung Kwak, ${ }^{a}$ Bong Kyu Yoo, ${ }^{a}$ Chul Soon Yong, $,{ }^{*}, a$ and \\ Han-Gon $\mathrm{CHOI}^{*, b}$ \\ ${ }^{a}$ College of Pharmacy, Yeungnam University; 214-1 Dae-Dong, Gyungsan, Gyungbuk 712-749, South Korea: and \\ ${ }^{b}$ College of Pharmacy, Hanyang University; 1271 Sa-3-Dong, Ansan 426-791, South Korea. \\ Received October 18, 2010; accepted April 24, 2011; published online May 23, 2011
}

In this study, a novel liquid self-emulsifying drug delivery system (SEDDS) containing curcumin was formulated and further developed into a solid form by a spray drying method using Aerosil 200 as the solid carrier. The optimum liquid SEDDS consisted of Lauroglycol Fcc, Labrasol and Transcutol HP as the oil phase, the surfactant and the co-surfactant at a weight ratio of $15.0: 70.8: 14.2(\mathrm{w} / \mathrm{w} / \mathrm{w})$, respectively. There was no difference in droplet size between the emulsions obtained from the liquid and solid forms of SEDDS. Solid state characterization of the solid SEDDS was performed by scanning electron micrograph (SEM), differential scanning calorimetry (DSC), and X-ray powder diffraction (XRPD). The drug formulated in the solid SEDDS was quickly and completely dissolved within $5 \mathrm{~min}$, both in $0.1 \mathrm{~N} \mathrm{HCl}$ and phosphate buffer $\mathrm{pH} 6.8$ dissolution media, whereas crude curcumin powder was significantly less dissoluble. The solid SEDDS formulation was stable for at least 3 months at $40^{\circ} \mathrm{C}$ with $75 \%$ relative humidity. After oral administration to rats, curcumin in the solid SEDDS resulted in significant improvement in in vivo absorption compared with that of curcumin powder. As the dose of curcumin formulated in solid SEDDS increased from 25 to $100 \mathrm{mg} / \mathrm{kg}$, the $C_{\max }$ and area under the drug concentration time curve $(A U C)$ of curcumin were increased by 4.6 and 7.6 times, respectively. However, the over-proportional increase in the $A U C$ in the higher dose group might be due to underestimation of $A U C$ in the lower dose group. In conclusion, this solid SEDDS is a promising solid dosage form for poorly water-soluble curcumin.

Key words curcumin; solid self-emulsifying; solubility; oral bioavailability

Curcumin (diferuloylmethane) is a yellow coloured phenolic substance derived from the spice herb Curcuma longa, usually called turmeric, which has beneficial activities including anti-inflammatory, antioxidant and anticancer activities. ${ }^{1,2)}$ Many clinical trials involving curcumin are currently ongoing. ${ }^{3)}$ However,the clinical advancement of this promising natural compound is hampered by its poor water solubility and short biological half-life, resulting in low bioavailability in both plasma and tissues. $\left.{ }^{4}{ }^{6}\right)$ The oral bioavailability of curcumin is very low (only $1 \%$ in rats). ${ }^{7,8)}$ In order to overcome these limitations, several approaches have been attempted including the combination of curcumin with adjuvants such as piperine. ${ }^{9}$ and the development of delivery vehicles consisting of liposomes, ${ }^{10)}$ nanoparticles ${ }^{11)}$ a phospholipid complex, ${ }^{12,13)}$ and the synthesis of structural analogues of curcumin. ${ }^{14}$

In recent years, lipid microemulsion formulations, with a particular emphasis on self-microemulsifying or self-emulsifying drug delivery systems, have been considered as an ideal alternative for improving the oral bioavailability of poorly water-soluble drugs. ${ }^{15}$ ) However, more recently, the concept of a solid self-emulsifying drug delivery system (SEDDS), which transfers a liquid self-emulsifying formulation into a solid dosage form, has been preferred by researchers. This novel drug delivery system combines the advantages of liquid SEDDS with those of a solid dosage form and overcomes the limitations associated with liquid formulations, such as a manufacturing process which results in high production costs and the incompatibility between the fill with the shells of the soft gelatin and storage temperatures. ${ }^{16}$ ) Unfortunately, very limited numbers of publications reporting such attempts are available. ${ }^{17-20)}$
Even though lipid microemulsion formulation has been evaluated for the enhancement of oral absorption of curcumin, ${ }^{21}$ this drug delivery system was prepared in the form of liquid SEDDS with Emulsifier OP : Cremorphor EL $(1: 1)$ as the surfactant, polyethylene glycol (PEG) 400 as the cosurfactant and ethyl oleate as the oil phase. Furthermore, this type of formulation inevitably suffered from the disadvantages described above. Therefore, the objectives of this study were to develop a novel liquid SEDDS with a totally different formulation and then to prepare a solid SEDDS of curcumin by using a spray-drying technique with Aerosil 200 as the inert solid carrier. This curcumin-loaded SEDDS was characterized by scanning electron microscopy (SEM), differential scanning calorimetry (DSC) and X-ray powder diffraction (XRPD). The dissolution study was performed in comparison with curcumin crude powder. Furthermore, a comparative study of bioavailability in rats was carried out to gain further insights into the absorption potential of curcumin via the SEDDS.

\section{MATERIALS AND METHODS}

Materials Curcumin was purchased from the SigmaAldrich Corp. (St. Louis, MO, U.S.A.). Polyglycolysed glycerides (Capryol 90, Labrafac CC, Labrafac Lipophile WL 1349, Labrasol, Labrafil M 1944 CS, Labrafac WL 2609 BS, Lauroglycol FCC, Peceol and Transcutol HP) were obtained from Gattefosse (Saint-Priest, Cedex, France). Corn oil, sesame oil, soybean oil and Cremorphor EL were supplied by Sigma (St. Louis, MO, U.S.A.). Tween 80 and PEG 400 were purchased from DC Chemical Co. (Seoul, South Korea). All other chemicals and solvents were of reagent grade and were 
used without further purification.

Solubility Study The solubility of curcumin in various oils, surfactants, and co-surfactants was determined. Two milliliters of each of the selected vehicles was added to each cap vial containing an excess of curcumin ( $c a .500 \mathrm{mg}$ ). After sealing, the mixture was heated at $40{ }^{\circ} \mathrm{C}$ in a water bath to facilitate solubilization using a vortex mixer. These mixtures were shaken at $25^{\circ} \mathrm{C}$ for $48 \mathrm{~h}$. After reaching equilibrium, each vial was centrifuged at $12500 \mathrm{~g}$ for $10 \mathrm{~min}$. The supernatant was filtered through a $0.45 \mu \mathrm{m}$ syringe filter membrane and diluted with methanol for quantification using an HPLC method on a LaChrom Elite HPLC System (Hitachi, Japan). An Inertsil ODS-3 $\mathrm{C}_{18}$ column $(4.6 \times 150 \mathrm{~mm}$, $5 \mu \mathrm{m})$ was used. The column temperature was held at $35^{\circ} \mathrm{C}$. Acetonitrile-2\% acetic glacial solution $(65: 35, \mathrm{v} / \mathrm{v})$ was used as the mobile phase at a flow rate of $1.0 \mathrm{ml} / \mathrm{min}$ with a run time of $12 \mathrm{~min}$. The injection volume was $20 \mu \mathrm{l}$ and UV detection was set at $425 \mathrm{~nm}$. Solubility studies were carried out in triplicate.

Pseudo-Ternary Phase Diagram Study Pseudo-ternary phase diagrams were constructed in order to obtain the concentration range of components for the existing region that could self-emulsify under dilution and gentle agitation. In brief, a series of SEDDS were prepared with varying concentrations of Lauroglycol Fcc (5-40\% w/w), Labrasol (20$90 \% \mathrm{w} / \mathrm{w})$ and Transcutol HP $(0-75 \% \mathrm{w} / \mathrm{w})$. Then, $0.2 \mathrm{ml}$ of each formulation was added in a drop-wise manner to a beaker containing $300 \mathrm{ml}$ water at room temperature $\left(37^{\circ} \mathrm{C}\right)$ and the contents were gently mixed with a magnetic stirring bar. The progress of the droplets was observed, particularly in view of their tendency to emulsify 'spontaneously'; when droplets were observed to spread easily in the water, forming a fine milky emulsion, this was judged as 'good,' and then the corresponding oil, surfactant and co-surfactant contents at appropriate weight ratios were selected.

Preparation of Curcumin-Loaded SEDDS The formulations were prepared by dissolving curcumin in mixtures with variable proportions of Lauroglycol Fcc (oil), Labrasol (surfactant) and Transcutol HP (co-surfactant). The level of curcumin was constant $(4.67 \% \mathrm{w} / \mathrm{w}$ of the vehicle) in all of the formulations. The components were added to a $10 \mathrm{ml}$ screw-capped glass tube and mixed by gentle stirring until a clear solution was obtained. Based on the results of the above experiment, the formulations were further optimized within the concentration scopes of the three ingredients forming the SEDDS. The weight ratio of surfactant to co-surfactant varied from $3: 1$ to $7: 1$, whereas the percentage of the oil phase was confined within a range of $5-25 \%$.

Preparation of Solid Curcumin-Loaded SEDDS Aerosil $200(1000 \mathrm{mg}$ ) was suspended in $150 \mathrm{ml}$ ethanol by sonication for at least $10 \mathrm{~min}$, and about $3.23 \mathrm{~g}$ of the optimized liquid SEDDS containing $225 \mathrm{mg}$ curcumin $(75 \mathrm{mg}$ curcumin was used for $1 \mathrm{~g}$ of blank optimized SEDDS system) was added with constant stirring until a good suspension was obtained. The suspension was then spray dried with a Buchi mini spray dryer B-190 apparatus (Buchi, Switzerland) under the following conditions: inlet temperature, $60{ }^{\circ} \mathrm{C}$; outlet temperature, $35^{\circ} \mathrm{C}$; aspiration, $85 \%$; feeding rate of the suspension, $5 \mathrm{ml} / \mathrm{min}$. The final drug content of the solid SEDDS was $5.33 \% \mathrm{w} / \mathrm{w}$ ratio.

Characterization of the Solid SEDDS. Reconstitution
In a $30 \mathrm{ml}$ vial, $100 \mu \mathrm{l}$ of liquid SEDDS and $125 \mathrm{mg}$ of the dry powders were dispersed in $15 \mathrm{ml}$ distilled water by vortex mixing for $10 \mathrm{~s}$, respectively. The resulting emulsion was incubated for $30 \mathrm{~min}$ at $25^{\circ} \mathrm{C}$ before samples were withdrawn for droplet size determination.

Droplet Size of Emulsions The size distribution of the droplets was determined by the Zetasizer Nano ZS (Malvern Instruments, Malvern, U.K.) dynamic light scattering particle size analyser at a wavelength of $635 \mathrm{~nm}$ and at a scattering angle of $90^{\circ}$ at $25^{\circ} \mathrm{C}$. The $z$-average diameter, also referred to as the harmonic intensity-weighted average hydrodynamic diameter, of the emulsions was derived from cumulated analysis by the Automeasure software (Malvern Instruments, Malvern, U.K.). All studies were repeated three times.

Scanning Electron Microscopy The morphological features of solid SEDDS were characterized further using a scanning electron microscope (S-400, Hitachi, Japan). The samples were fixed on a brass specimen club using doublesided sticky tape and made electrically conductive by coating in a vacuum $(6 \mathrm{~Pa})$ with platinum $(6 \mathrm{~nm} / \mathrm{min})$ using a Hitachi Iron Sputter (E-1030) for $30 \mathrm{~s}$ at $15 \mathrm{~mA}$.

Differential Scanning Calorimetry The thermochemical properties of the solid SEDDS were characterized by differential scanning calorimetry (DSC) thermogram analysis (DSC Q200, TA Instruments, U.S.A.). After weighing about $2 \mathrm{mg}$ of a test sample using a microbalance (Satorius), the sample was placed into an aluminium pan with a lid and the pan was sealed. An empty aluminium pan with its lid was used as a control. The samples were purged with pure dry nitrogen at a flow rate of $70 \mathrm{ml} / \mathrm{min}$. The temperature ramp speed was set at $10^{\circ} \mathrm{C} / \mathrm{min}$ and the heat flow was recorded from 30 to $230^{\circ} \mathrm{C}$. The temperature scale was calibrated with high purity standards.

X-Ray Powder Diffraction The physical states of the pure curcumin, silicon dioxide, solid SEDDS as well as physical mixture prepared by mixing well $1000 \mathrm{mg}$ of Aerosil 200 and $225 \mathrm{mg}$ of curcumin using mortar and pestle, were evaluated by X-ray powder diffraction (XRPD). Diffraction patterns were obtained using a BRUKER D8 FOCUS High Resolution Powder Diffractometer (BRUKER AXS, Germany) equipped with a scintillation counter detector and a divergent beam. This beam employed a $\mathrm{Cu} K \alpha$ radiation source with a wavelength of $\lambda=1.5418$ containing $2 \mathrm{~mm}$ slits over a range of $10-50^{\circ} 2-\theta$. X-Ray diffraction data were collected at room temperature and scanned with a step size of $5^{\circ} 2-\theta$ and a dwell time of $12 \mathrm{~min}$ at each step.

Dissolution The curcumin-loaded solid SEDDS and the powder equivalent of $10 \mathrm{mg}$ curcumin were each placed in a dissolution tester (Shinseang Instrument Co., South Korea). The dissolution test was performed at $36.5^{\circ} \mathrm{C}$ using the paddle method at $100 \mathrm{rpm}$ with $900 \mathrm{ml}$ of $0.1 \mathrm{~N} \mathrm{HCl}$ and phosphate buffer $\mathrm{pH} 6.8$ as dissolution media. At a predetermined interval, $3 \mathrm{ml}$ of the medium was sampled and filtered through a membrane filter $(0.45 \mu \mathrm{m})$. The concentration of curcumin in the resulting solution $(10 \mu \mathrm{l})$ was analysed using the HPLC method as described above.

Stability Studies To evaluate the chemical and physical stability of curcumin-loaded solid SEDDS, the formulation was placed in the glass vial protected from light with or without rubber-stopper at $40{ }^{\circ} \mathrm{C}$ with $75 \%$ relative humidity for 3 months. Samples were removed at 0 and $90 \mathrm{~d}$ of interval 
Table 1. Solubility of Curcumin in Various Vehicles

\begin{tabular}{lc|lc}
\hline \hline \multicolumn{1}{c}{ Vehicle } & $\begin{array}{c}\text { Solubility } \\
(\mathrm{mg} / \mathrm{ml})\end{array}$ & \multicolumn{1}{c}{ Vehicle } & $\begin{array}{c}\text { Solubility } \\
(\mathrm{mg} / \mathrm{ml})\end{array}$ \\
\hline Tween-80 & $34.38 \pm 0.78$ & Corn oil & $1.48 \pm 0.06$ \\
Labrasol & $52.15 \pm 1.67$ & Sesame oil & $0.97 \pm 0.02$ \\
Trancutol HP & $112.98 \pm 17.40$ & Peceol & $1.67 \pm 0.09$ \\
PEG 400 & $35.58 \pm 3.83$ & Soybean oil & $0.95 \pm 0.03$ \\
Cremorphor EL & $37.04 \pm 7.86$ & Ethyl oleate & $0.43 \pm 0.02$ \\
Capryol 90 & $7.75 \pm 0.11$ & Labrafac CC & $2.62 \pm 0.08$ \\
Lauroglycol Fcc & $8.20 \pm 0.08$ & Labrafac lipophile WL 1349 & $2.63 \pm 0.03$ \\
Labrafil M 1944cs & $4.11 \pm 0.07$ & Labrafac WL 2609 BS & $4.43 \pm 0.11$ \\
\hline
\end{tabular}

Each value represents the mean \pm S.D. $(n=3)$.

and checked for droplet size, curcumin content, and \% dissolution at 5 min using the methods described above.

In Vivo Absorption Study Male Sprague-Dawley rats weighing 250 $\pm 20 \mathrm{~g}$ were purchased from Charles River Co., Korea (Orient, Seoul, South Korea). Eighteen rats were randomly divided into three groups and fasted for $10-12 \mathrm{~h}$ prior to the experiments, although they were allowed free access to water. All animal care and procedures were conducted according to the Guiding Principles in the Use of Animals in Toxicology, as adopted in 1989, revised in 1999 and amended in 2008 by the society of toxicology (SOT, 2008). Furthermore, the protocols for the animal studies were approved by the Institute of Laboratory Animal Resources of Yeungnam University.

Each rat, anaesthetized in an ether-saturated chamber, was secured on a surgical board in the supine position with a thread. A polyethylene tube was inserted into the right femoral artery of the rat. The rats in the first group were orally administered $1 \mathrm{ml}$ of $0.5 \%$ carboxymethyl cellulose (CMC) aqueous suspension containing curcumin at a dose of $100 \mathrm{mg} / \mathrm{kg}$, and those in the remaining two groups received curcumin SEDDS re-dispersed in $1 \mathrm{ml}$ of distilled water at doses of 25 and $100 \mathrm{mg} / \mathrm{kg}$ (as curcumin), respectively. Then, $0.3 \mathrm{ml}$ of blood was collected from the right femoral artery at predetermined time intervals and centrifuged at $10000 \boldsymbol{g}$ for 10 min using a $5415 \mathrm{C}$ centrifuge (Eppendorf, U.S.A.).

Plasma $(150 \mu \mathrm{l})$ was mixed with $30 \mu \mathrm{l}$ methanol solution containing indomethacin $(3 \mathrm{mg} / \mathrm{ml})$ as an internal standard. The plasma was then extracted twice with $1.5 \mathrm{ml}$ of ethyl acetate by vigorous mixing for $5 \mathrm{~min}$. After centrifugation at $11000 \mathrm{~g}$ for $4 \mathrm{~min}$, the upper organic layer was removed and placed into a clean micro-centrifuge tube. The combined organic phase was then evaporated at $40{ }^{\circ} \mathrm{C}$ in a heated centrifugal evaporator (EYELA CVE-200D; Tokyo Rikakikai Co., Tokyo, Japan). The residue was reconstituted with $100 \mu \mathrm{l}$ of the mobile phase, vortexed for $5 \mathrm{~min}$ and centrifuged again for $5 \mathrm{~min}$ at $11000 \mathrm{~g}$. Then, $50 \mu \mathrm{l}$ of the supernatant was analysed by HPLC, as mentioned above.

Plasma concentration of curcumin below lower limit of quantification $(10 \mathrm{ng} / \mathrm{ml})$ was reported as zero. All data are expressed as means \pm standard deviation (S.D.) or medians (ranges). Levels of statistical significance $(p<0.05)$ were assessed using the Students $t$-test between two means for the unpaired data.

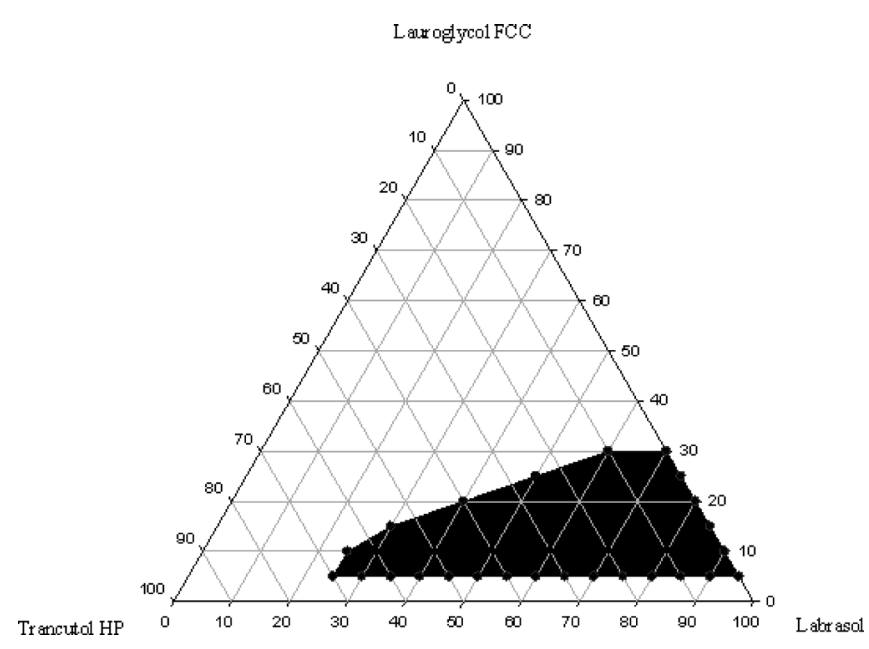

Fig. 1. Phase Diagram of the SEDDS Composed of Lauroglycol Fcc, Labrasol and Transcutol HP, the Shadow Area Represents o/w Microemulsion Region

\section{RESULTS AND DISCUSSION}

Solubility Study Self-emulsifying formulations consisting of oil, a surfactant, a co-surfactant and a drug should be clear and homogeneous liquids at ambient temperature. Therefore, the components used in the system should have the maximal solubilizing potential for the drug to achieve optimum drug loading while maintaining an excellent emulsifying performance. The solubility of curcumin in various oils, surfactants and co-surfactants is shown in Table 1. Of the oils tested, Lauroglycol Fcc gave highest solubility for curcumin, followed by Capryol 90 and Labrafac WL 2609 BS. Thus, Lauroglycol Fcc was selected as the oil phase for further investigations. Among the various surfactants screened, Labrasol exhibited a good solubilizing potential for curcumin, while out of the various co-surfactants Transcutol HP exhibited the highest solubility for curcumin. Mixing Transcutol P with Labrasol generally helps to improve the emulsifying ability of Labrasol. ${ }^{22)}$ Furthermore, both Labrasol and Transcutol HP were previously shown to play roles as bioavailability enhancers. ${ }^{23)}$ Therefore, Labrasol and Transcutol HP were chosen as the surfactant and co-surfactant, respectively, for further investigations.

Construction of Phase Diagrams Pseudo-ternary phase diagrams were constructed to obtain appropriate concentration ranges of components for forming fine emulsions. The pseudo-ternary phase diagram of the system containing the mixture of Lauroglycol Fcc, Labrasol and Transcutol HP is shown in Fig. 1. It was observed that the concentration of the oil phase should be less than $30 \% \mathrm{w} / \mathrm{w}$ in order to generate spontaneous emulsion formation. Moreover, the emulsification performance was not good when the surfactant concentration was below $25 \% \mathrm{w} / \mathrm{w}$, since the formulations surrounding the good self-emulsifying region in the phase diagram exhibited a poor emulsion-forming ability.

Formulation Optimization of Curcumin-Loaded Liquid SEDDS The droplet size of an emulsion is a crucial factor in self-emulsification performance because it determines the rate and extent of drug release as well as absorption. Thus, the formulation of SEDDS with $4.67 \% \mathrm{w} / \mathrm{w}$ drug loading was further optimized using droplet size as a key at- 


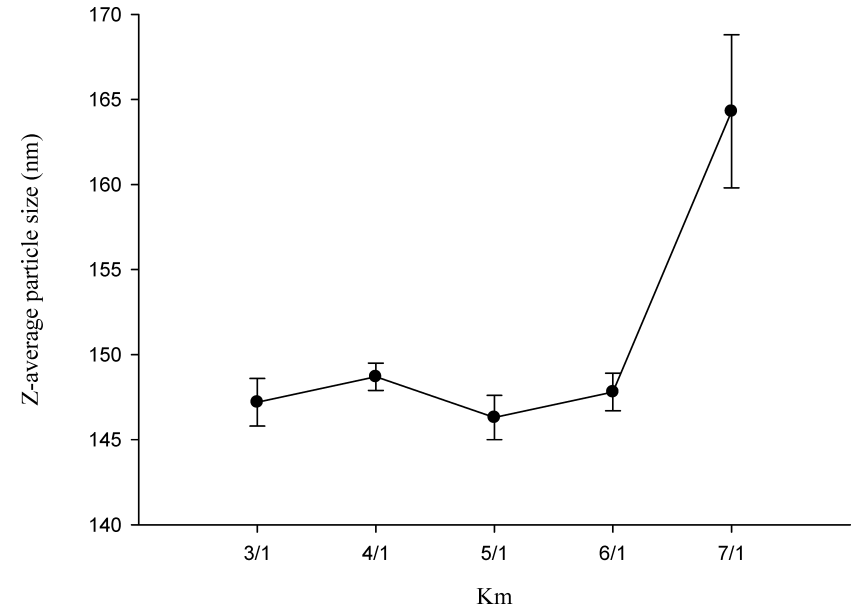

Fig. 2. Effect of $K_{\mathrm{m}}$ on the Droplet Size of the Emulsion Formed in Water $K_{\mathrm{m}}=$ the weight ratio of surfactant to co-surfactant to oil phase $=10 \%(\mathrm{w} / \mathrm{w})$. Each value represents the mean \pm S.D. $(n=3)$.

Table 2. Composition of the Optimized Liquid SEDDS Formulation

\begin{tabular}{ccl}
\hline \hline Vehicle & Percentage $(w / w)$ & Role \\
\hline Lauroglycol Fcc & 15.0 & Oil phase \\
Labrasol & 70.8 & Surfactant \\
Transcutol HP & 14.2 & Co-surfactant \\
\hline
\end{tabular}

tribute. As shown in Figs. 2 and 3, when the oil phase in the system was set at a level of $10 \%$, the mean $z$-average diameter of the emulsion formed did not significantly change as the weight ratio of surfactant to co-surfactant $\left(K_{\mathrm{m}}\right)$ increased from $3: 1$ to $6: 1$, after which a significant increase in the $z$-average diameter was observed. Among the tested $K_{\mathrm{m}}$, a weight ratio of $5: 1$ resulted in the smallest droplet size. On the other hand, the mean $z$-average diameter of the emulsion decreased to $136.5 \mathrm{~nm}$ when the oil phase was increased from 5 to $15 \%$, after which the mean $z$-average diameter of the emulsion increased slightly. As a result, an optimized system containing a $15 \%$ oil phase with a $K_{\mathrm{m}}$ of $5: 1$ was obtained. The details of the formulation are shown in Table 2.

Reconstitution Properties of the Solid SEDDS In the present study, Aerosil 200 was used as carrier to prepare solid SEDDS due to its well-known inert properties and high oil absorption capacity. The $z$-average diameters and polydispersity indexes for the solid and liquid SEDDS are presented in Table 3. As shown in this table, the $z$-average droplet sizes for liquid SEDDS and solid SEDDS systems were 143.4 and $147.3 \mathrm{~nm}$, respectively. Both the mean droplet size and size distribution (polydispersity index) of the emulsion remained almost unchanged in spite of the conversion from a liquid to a solid state, indicating that the spray-drying process did not have a remarkable influence on the emulsification performance. The typical emulsion droplet size distribution before and after spray drying (Figs. 4A, B) also revealed a comparable self-emulsification performance between the liquid and the solid SEDDS.

Solid State Characterization of Solid SEDDS Figure 5 shows the scanning electron micrographs of Aerosil 200, the crude curcumin powder and the solid SEDDS. Aerosil 200

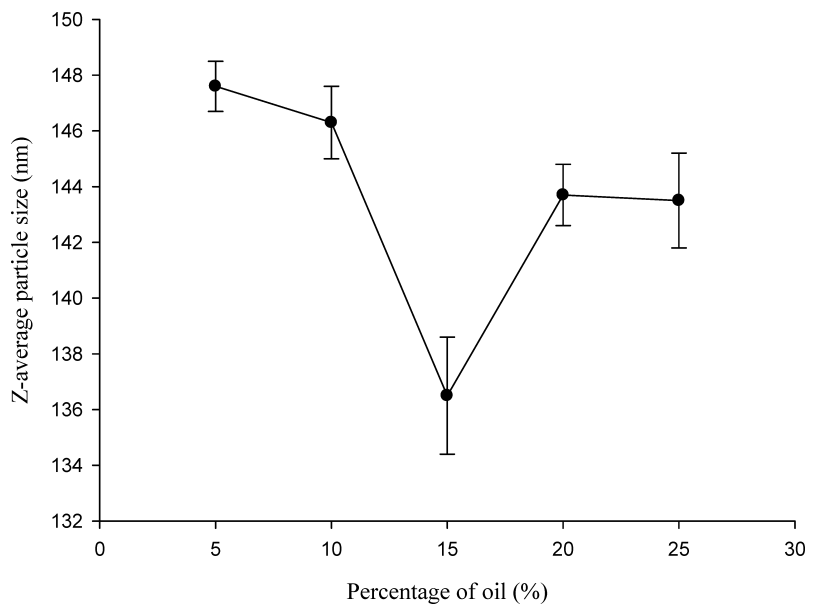

Fig. 3. Effect of Oil Phase Percentage on the Droplet Size of the Emulsion Formed in Water

$K_{\mathrm{m}}=5: 1(\mathrm{w} / \mathrm{w})$. Each value represents the mean \pm S.D. $(n=3)$.

Table 3. Mean Emulsion Droplet Sizes and Polydispersity Indexes (PDI) of the Liquid and Solid SEDDS

\begin{tabular}{lcc}
\hline \hline Formulation & $z$-Average diameter $(\mathrm{nm})$ & PDI \\
\hline Liquid SEDDS & $143.4 \pm 3.7$ & $0.257 \pm 0.038$ \\
Solid SEDDS & $147.3 \pm 5.8$ & $0.289 \pm 0.021$ \\
\hline
\end{tabular}

Each value represents the mean \pm S.D. $(n=3)$.

(Fig. 5A) appeared as a rough surface with porous particles. The curcumin powder (Fig. 5B) appeared with a rectangular crystalline shape with a smooth surface, its particle size varied in a wide range from less than $1 \mu \mathrm{m}$ to larger than dozens of micrometers. The image of the solid SEDDS containing curcumin (Fig. 5C), however, illustrate that the particles had the same outer macroscopic morphology consisting of aggregated spherical particles with relatively deep dents and similar diameters $(2-15 \mu \mathrm{m})$. Following spray drying, the crystalline curcumin turned out to be highly amorphous in nature.

The physical state of curcumin in the solid SEDDS was investigated since it would have an important influence on the in vitro and in vivo release characteristics. X-Ray diffraction (XRD) provided information on the crystallinity and crystal orientation, and the shape of the XRD patterns reflected the state of the regular arrangement of molecules inside the crystals. Many diffraction peaks of a high intensity were observed in the diffraction pattern of the raw curcumin material due to its crystallinity (Fig. 6A). Furthermore, all of the major characteristic crystalline peaks that appeared in the drug were also observed in the physical mixture (Fig. 6B). On the other hand, the solid SEDDS (Fig. 6D) showed similar characteristics to the Aerosil 200 pattern (Fig. 6C), in which none of the diffraction peaks were observed and no peaks for crystalline curcumin were observed either.

Figure 7 shows the DSC curves of crude curcumin powder, Aerosil 200, the physical mixture and the solid SEDDS. The thermogram of pure curcumin (Fig. 7A) exhibited an endothermic peak at about $180^{\circ} \mathrm{C}$, corresponding to its melting point. Aerosil 200 showed no specific peaks from 30 to $250{ }^{\circ} \mathrm{C}$ (Fig. 7D). However, a melting endotherm having dou- 
(A)

Size Distribution by Intensity

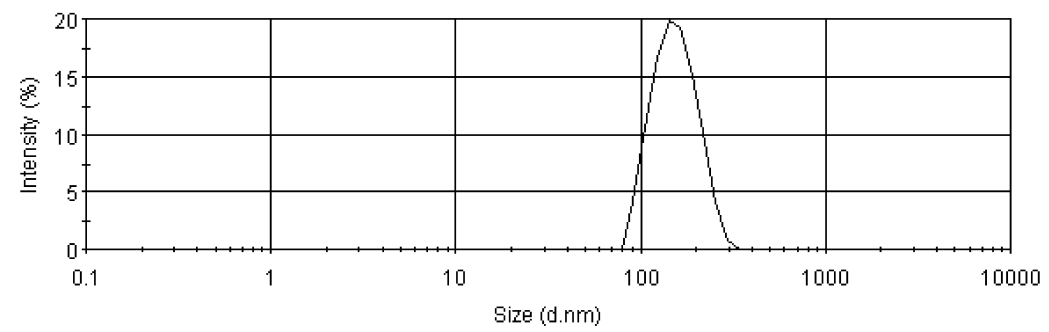

(B)

Size Distribution by Intensity

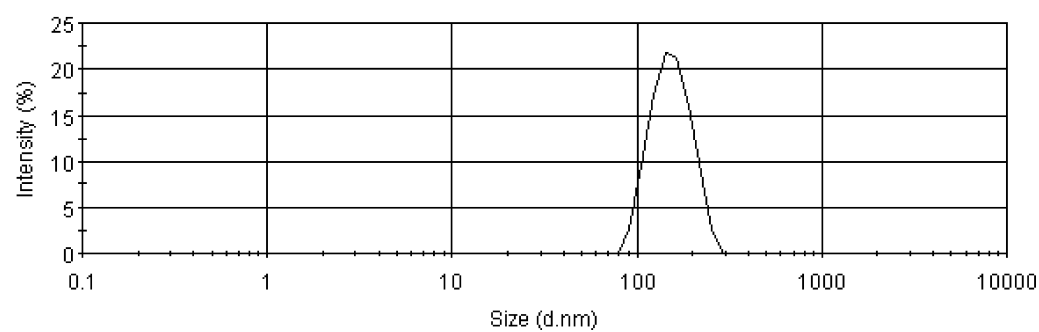

Fig. 4. Emulsion Droplet Size Distribution

(A) Liquid SEDDS, (B) solid SEDDS.
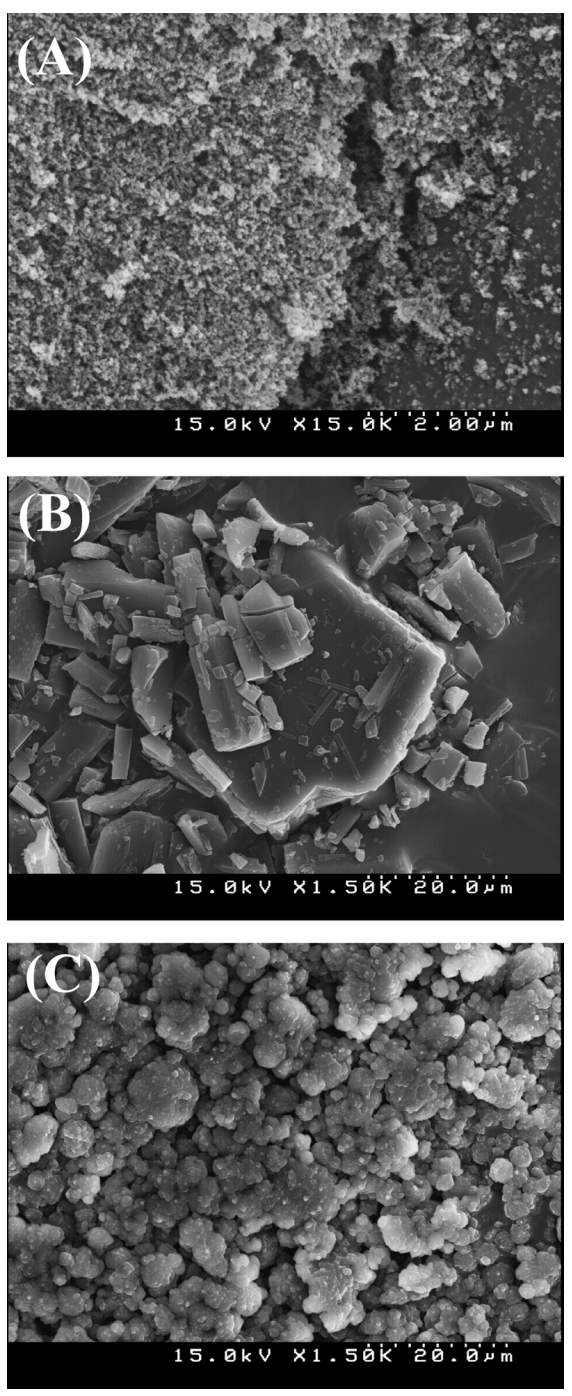

Fig. 5. Scanning Electron Micrographs $(\times 1500)$

(A) Aerosil 200, (B) curcumin powder, (C) solid SEDDS.

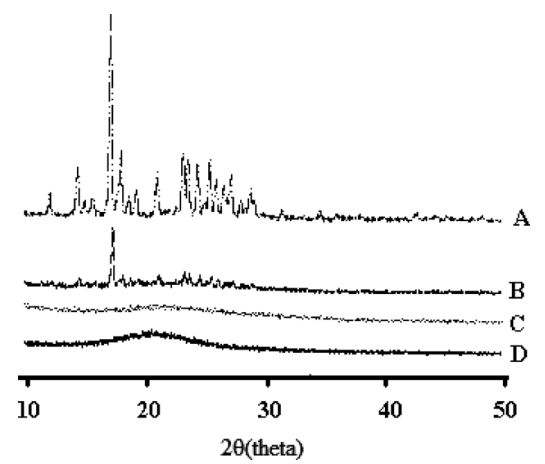

Fig. 6. X-Ray Powder Diffraction

(A) Curcumin powder, (B) physical mixture, (C) Aerosil 200, (D) solid SEDDS.

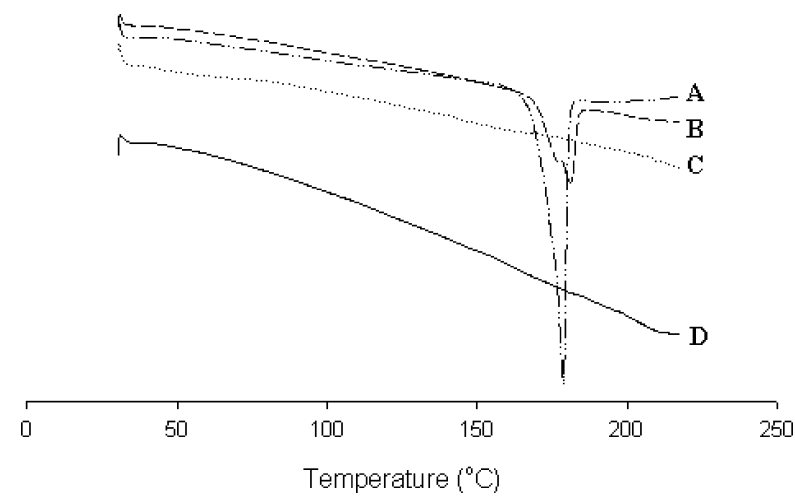

Fig. 7. Differential Scanning Calorimetric Thermogram

(A) Curcumin powder, (B) physical mixture, (C) solid SEDDS, (D) Aerosil 200.

ble peaks shape with reduced intensity was observed in the physical mixture (Fig. 7B). This phenomenon might be due to the uneven distribution of curcumin crystals between inside and outside of small pores on the surface of Aerosil 200, as a result, curcumin crystals included in small pores caused 


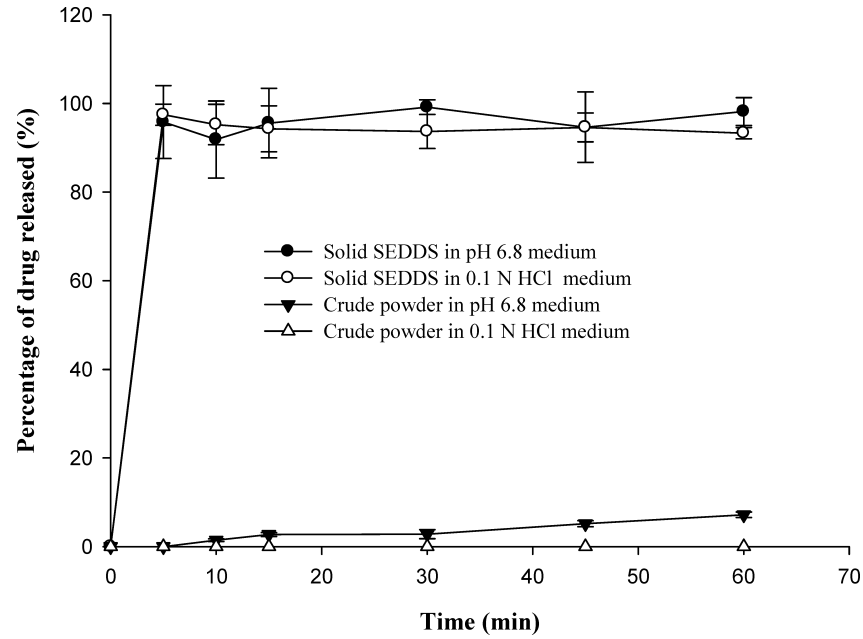

Fig. 8. Dissolution Profiles of Curcumin Powder and Curcumin-Loaded SEDDS in $0.1 \mathrm{~N} \mathrm{HCl}$ and Phosphate Buffer pH 6.8

Each value represents the mean \pm S.D. $(n=6)$

Table 4. Evaluation Data of Solid SEDDS Formulation Subjected to Stability Studies

\begin{tabular}{|c|c|c|c|}
\hline \multirow{3}{*}{ Parameters } & \multicolumn{3}{|c|}{ Time in day } \\
\hline & \multirow{2}{*}{0} & \multicolumn{2}{|c|}{90} \\
\hline & & Open & Closed \\
\hline Droplet size $(\mathrm{nm})^{a)}$ & $147.3 \pm 5.8$ & $156.3 \pm 3.9$ & $162.8 \pm 2.7$ \\
\hline Polydispersity index ${ }^{a)}$ & $0.289 \pm 0.021$ & $0.263 \pm 0.038$ & $0.299 \pm 0.009$ \\
\hline$\%$ drug content $t^{a}$ & $5.21 \pm 0.22$ & $5.18 \pm 0.09$ & $5.31 \pm 0.13$ \\
\hline$\%$ dissolution at $5 \mathrm{~min}(\mathrm{pH} 6.8)^{b)}$ & $95.8 \pm 8.2$ & $96.4 \pm 3.5$ & $97.2 \pm 4.3$ \\
\hline$\%$ dissolution at $5 \mathrm{~min}(\mathrm{pH} 1.2)^{b)}$ & $97.5 \pm 2.4$ & $95.5 \pm 4.3$ & $93.8 \pm 3.5$ \\
\hline
\end{tabular}

Each value represents the mean \pm S.D. $a$ ) $n=3 ; b) n=6$.

slight melting point depression from the original melting point. In the case of curcumin solid SEDDS, the endothermic peak of the drug was absent (Fig. 7C). Therefore, it could be concluded that curcumin in the solid SEDDS was in the amorphous form after the fabrication. It is known that transforming the physical state of a drug to the amorphous or partially amorphous state leads to a high-energy state and high disorder, resulting in enhanced solubility. As a result, it was expected that the solid particles would also have enhanced solubility.

In Vitro Dissolution Test As shown in Fig. 8, for the crude powder of curcumin, only less than $7 \%$ of the drug was dissolved in the phosphate buffer $\mathrm{pH} 6.8$ medium, and no drug dissolution was observed in the $0.1 \mathrm{~N} \mathrm{HCl}$ medium during the period of determination. This dissolution characteristic of crude curcumin powder in different media may be a result of its intrinsically weak acidity. However, the drug dissolution profiles of curcumin-loaded solid SEDDS in $0.1 \mathrm{~N} \mathrm{HCl}$ and phosphate buffer $\mathrm{pH} 6.8$ were not significantly different: both showed complete dissolution within $5 \mathrm{~min}$ in spite of the $\mathrm{pH}$ level of the aqueous media. Following self-emulsification in the dissolution media, the SEDDS provides ultra low interfacial tensions and large $\mathrm{o} / \mathrm{w}$ interfacial areas, leading to the incorporation of poorly water-soluble pharmaceuticals inside the fine oil droplets. Therefore, the SEDDS has an advantage

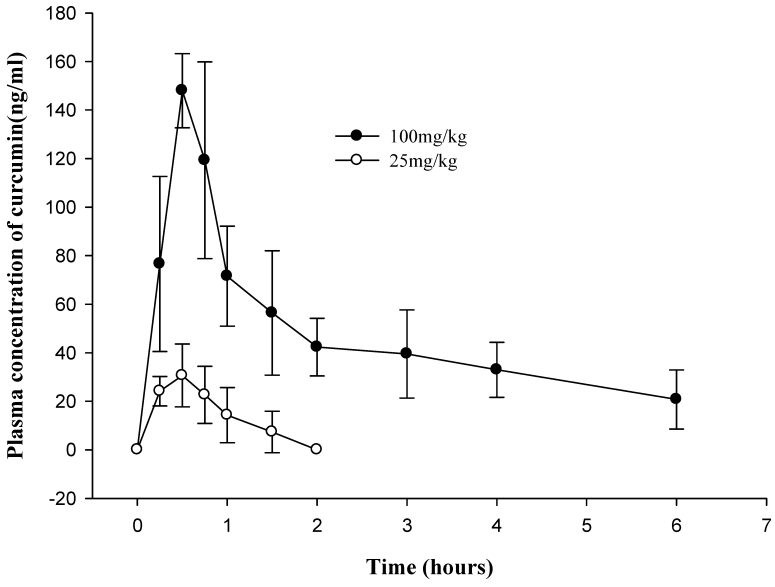

Fig. 9. Plasma Concentration-Time Profiles of Curcumin Following the Oral Administration of Two Doses of Curcumin SEDDS to Rats (Equivalent to 25 and $100 \mathrm{mg} / \mathrm{kg}$ Curcumin, Respectively)

The plasma concentrations of drug after oral administration of curcumin powder were not detected due to its very small absorption in rats. Each value represents the mean \pm S.D. $(n=6)$.

Table 5. Pharmacokinetic Parameters after Oral Administration of Curcumin-Loaded Solid SEDDS to Rats

\begin{tabular}{lcc}
\hline \hline \multicolumn{1}{c}{ Parameters } & $25 \mathrm{mg} / \mathrm{kg}$ & $100 \mathrm{mg} / \mathrm{kg}$ \\
\hline$A U C(\mathrm{~h} \cdot \mathrm{ng} / \mathrm{ml})$ & $37.05 \pm 17.73$ & $282.54 \pm 61.37$ \\
$C_{\max }(\mathrm{ng} / \mathrm{ml})$ & $33.97 \pm 9.84$ & $155.56 \pm 18.34$ \\
$T_{\max }(\mathrm{h})$ & $0.54 \pm 0.10$ & $0.58 \pm 0.13$ \\
$t_{1 / 2}(\mathrm{~h})$ & $\left.\mathrm{NA}^{a}\right)$ & $2.45 \pm 0.90$ \\
$K_{\mathrm{el}}\left(\mathrm{h}^{-1}\right)$ & $\mathrm{NA}^{a}$ & $0.30 \pm 0.10$ \\
\hline
\end{tabular}

Each value represents the mean \pm S.D. $(n=6)$. The plasma concentrations of the drug after the oral administration of curcumin powder were not detected due to its very low absorption in rats. $a$ ) Not applicable.

in possessing a high solubilization capacity which ensures a higher dissolution rate in comparison to crude powder. ${ }^{24)}$ In addition, the drug dissolution profiles of the liquid SEDDS showed no significant differences when compared to those of the solid SEDDS (data not shown), suggesting that the SEDDS preserves a similar performance in emulsification regardless of the form (i.e. liquid or solid).

Stability Studies Table 4 gives the results of the evaluation test conducted on stability samples. The solid SEDDS formulation was found to be stable for at least 3 months at $40{ }^{\circ} \mathrm{C}$ with $75 \%$ relative humidity. There was no significant change in mean droplet size and size distribution of the resultant emulsion, drug content, and drug release (at $5 \mathrm{~min}$ ) in both dissolution media. It was also seen that the spraying dried formulation was stable to humidity, as no significant difference was observed between the samples stored in open and closed with respect to evaluated parameters. Thus, these studies confirmed the stability of the developed formulation.

In Vivo Absorption Study Only solid curcumin-loaded SEDDS was chosen to be tested versus curcumin powder for the in vivo absorption study considering the fact that both liquid and solid SEDDS showed comparable self-emulsification properties, droplet sizes and drug release patterns. However, the total plasma concentrations of drug after oral administration of curcumin powder evan at a dose of $100 \mathrm{mg} / \mathrm{kg}$ could not be detected due to its very small absorption in rats. 
On the other hand, the plasma levels of curcumin in the solid SEDDS group at the lower dose level $(25 \mathrm{mg} / \mathrm{kg})$ remained detectable for up to $1.5 \mathrm{~h}$ after the oral dose. Thus, the higher plasma concentrations of curcumin in solid SEDDS were contributed by improving the solubility of drug. The profiles of the plasma concentration of curcumin versus time after the oral administration of curcumin-loaded solid SEDDS are depicted in Fig. 9. The corresponding pharmacokinetic parameters for these two groups are shown in Table 5. As the oral dose of curcumin was increased from 25 to $100 \mathrm{mg} / \mathrm{kg}$ by 4.0 times, the $C_{\max }$ was increased from $33.97 \pm 9.84$ to $155.56 \pm 18.34 \mathrm{ng} / \mathrm{ml}$ by 4.6 times similarly. However, in the case of $A U C$, it was increased from $37.05 \pm 17.73$ to $282.54 \pm 61.37 \mathrm{~h} \cdot \mathrm{ng} / \mathrm{ml}$ by 7.6 times, suggesting over-proportional increase in the $A U C$. It is worth noting that in the present study, the plasma concentrations of curcumin detectable but below lower limit of quantification $(10 \mathrm{ng} / \mathrm{ml})$ were observed mostly (especially after $1.5 \mathrm{~h}$ ) in the low dose group and they were reported as zero, the $A U C$ in this group was thus underestimated. In another word, this nonlinear increase in $A U C$ in the high dose group was most possibly due to the underestimation of $A U C$ in the low dose group. There was no significant difference in the time taken to reach the peak concentration $\left(T_{\max }\right)$ of curcumin between the low dose group $(0.54 \pm 0.10 \mathrm{~h})$ and the high dose group $(0.58 \pm 0.13 \mathrm{~h})$.

The oral bioavailabilities of many poorly water-soluble drugs have been improved by SEDDS employing a single or combined mechanism. One of the main reasons for the enhanced drug oral bioavailability by SEDDS is the excellent efficiency of SEDDS in improving the drug solubility and in increasing the dissolution rate. ${ }^{25,26)}$ Following self-emulsification in the GI tract after oral administration, SEDDS provides ultra low interfacial tensions and large o/w interfacial areas, resulting in the incorporation of poorly water-soluble pharmaceuticals inside the fine oil droplets. The large specific surface area of the fine oil droplets also enables a more efficient drug transport through the intestinal aqueous boundary layer, leading to an improvement in oral bioavailability. ${ }^{27)}$ In the present study, the results obtained from the in vitro dissolution test confirmed that curcumin had been quickly released from the solid SEDDS and completely in both the $0.1 \mathrm{~N} \mathrm{HCl}$ and phosphate buffer $\mathrm{pH} 6.8$ dissolution media, whereas crude curcumin was less dissoluble. Accordingly, oral curcumin absorption in the solid SEDDS was significantly increased compared to that of the suspension formulation. This situation is found for many poor solubility drugs such as 9-nitrocamptothecin and acyclovir. ${ }^{28,29)}$ The superior performance of curcumin SEDDS in oral absorption might also be attributed to increasing membrane fluidity and permeability in intestinal epithelial cells, ${ }^{30,31)}$ although this needs further confirmation.

\section{CONCLUSION}

In this study, a novel liquid SEDDS consisting of Lauroglycol FCC, Labrasol and Transcutol HP as an oil phase, a surfactant and a co-surfactant, respectively, was formulated and further developed into a solid SEDDS by a spray-drying technique using Aerosil 200 as the solid carrier. This solid SEDDS preserved the self-emulsification performance of the liquid SEDDS and gave a faster in vitro dissolution rate than the crude powder both in $0.1 \mathrm{~N} \mathrm{HCl}$ and phosphate buffer $\mathrm{pH}$ 6.8 dissolution media. Furthermore, it allowed a significant improvement in curcumin bioavailability in rats after oral administration compared to a powder suspension formulation. As the dose of curcumin formulated in solid SEDDS increased from 25 to $100 \mathrm{mg} / \mathrm{kg}$, the $C_{\max }$ and $A U C$ of curcumin were increased by 4.6 and 7.6 times, respectively. However, the over-proportional increase in the $A U C$ in the higher dose group might due to underestimation of $A U C$ in the lower dose group. Considering the limitations associated with liquid SEDDS, a solid powder formulation should be a more acceptable form. Furthermore, our results suggest that the solid SEDDS could be considered and further evaluated for the oral delivery of lipophilic poorly soluble drugs for which an oral route of administration is desirable.

\section{The authors declare no conflict of interest.}

Acknowledgements This work was supported by Basic Science Research Program through the National Research Foundation of Korea (NRF) funded by the Ministry of Education, Science and Technology (No. 2010-0024185) and was financially supported by the Ministry of Science and Technology (M10414030001-05N1403-00140) in South Korea.

\section{REFERENCES}

1) Araújo C. C., Leon L. L., Mem. Inst. Oswaldo Cruz, 96, 723-728 (2001).

2) Hsu C. H., Cheng A. L., Adv. Exp. Med. Biol., 595, $471-480$ (2007).

3) Teiten M. H., Eifes S., Dicato M., Diederich M., Toxins, 2, 128-162 (2010).

4) Cheng A. L., Hsu C. H., Lin J. K., Hsu M. M., Ho Y. F., Shen T. S., Ko J. Y., Lin J. T., Lin B. R., Ming-Shiang W., Yu H. S., Jee S. H., Chen G. S., Chen T. M., Chen C. A., Lai M. K., Pu Y. S., Pan M. H., Wang Y. J., Tsai C. C., Hsieh C. Y., Anticancer Res., 21, 2895-2900 (2001).

5) Anand P., Kunnumakkara A. B., Newman R. A., Aggarwal B. B., Mol. Pharm., 4, 807-818 (2007).

6) Burgos-Morón E., Calderón-Montaño J. M., Salvador J., Robles A., López-Lázaro M., Int. J. Cancer, 126, 1771-1775 (2010).

7) Pan M. H., Huang T. M., Lin J. K., Drug Metab. Dispos., 27, 486494 (1999).

8) Yang K. Y., Lin L. C., Tseng T. Y., Wang S. C., Tsai T. H., J. Chromatogr. B Analyt. Technol. Biomed. Life Sci., 853, 183-189 (2007).

9) Shoba G., Joy D., Joseph T., Majeed M., Rajendran R., Srinivas P. S., Planta Med., 64, 353-356 (1998).

10) Li L., Braiteh F. S., Kurzrock R., Cancer, 104, 1322-1331 (2005).

11) Shaikh J., Ankola D. D., Beniwal V., Singh D., Kumar M. N., Eur. J. Pharm. Sci., 37, 223-230 (2009).

12) Maiti K., Mukherjee K., Gantait A., Saha B. P., Mukherjee P. K., Int. J. Pharm., 330, 155-163 (2007).

13) Marczylo T. H., Verschoyle R. D., Cooke D. N., Morazzoni P., Steward W. P., Gescher A. J., Cancer Chemother. Pharmacol., 60, 171-177 (2007).

14) Mosley C. A., Liotta D. C., Snyder J. P., Adv. Exp. Med. Biol., 595, $77-103$ (2007).

15) Cui S. X., Nie S. F., Li L., Wang C. G., Pan W. S., Sun J. P., Biol. Pharm. Bull., 31, 118-125 (2008).

16) Tang B., Cheng G., Gu J. C., Xu C. H., Drug Discov. Today, 13, 606612 (2008).

17) Nazzal S., Khan M. A., Int. J. Pharm., 315, 110-121 (2006).

18) Franceschinis E., Voinovich D., Grassi M., Perissutti B., FilipovicGrcic J., Martinac A., Meriani-Merlo F., Int. J. Pharm., 291, 87-97 (2005).

19) Yi T., Wan J., Xu H., Yang X., Eur. J. Pharm. Biopharm., 70, 439444 (2008).

20) Balakrishnan P., Lee B. J., Oh D. H., Kim J. O., Hong M. J., Jee J. P., Kim J. A., Yoo B. K., Woo J. S., Yong C. S., Choi H. G., Eur. J. Pharm. Biopharm., 72, 539-545 (2009). 
21) Cui J., Yu B., Zhao Y., Zhu W., Li H., Lou H., Zhai G., Int. J. Pharm., 371, 148-155 (2009).

22) Kim H. J., Yoon K. A., Hahn M., Park E. S., Chi S. C., Drug Dev. Ind. Pharm., 26, 523-529 (2000).

23) Amit A. K., Vandana B. P., AAPS PharmSciTech, 9, 191-196 (2008).

24) Xi J., Chang Q., Chan C. K., Meng Z. Y., Wang G. N., Sun J. B., Wang Y. T., Tong H. H., Zheng Y., AAPS PharmSciTech, 10, 172-182 (2009).

25) Ghosh P. K., Murthy R. S., Curr. Drug Deliv., 3, 167-180 (2006).

26) Liu Y., Zhang P., Feng N., Zhang X., Wu S., Zhao J., Int. J. Pharm.,
365, 136-142 (2009).

27) Patel D., Sawant K. K., Curr. Drug Deliv., 6, 419-424 (2009).

28) Lu J. L., Wang J. C., Zhao S. X., Liu X. Y., Zhao H., Zhang X., Zhou S. F., Zhang Q., Eur. J. Pharm. Biopharm., 69, 899-907 (2008).

29) Ghosh P. K., Majithiya R. J., Umrethia M. L., Murthy R. S., AAPS PharmSciTech, 7, E172-E177 (2006).

30) Dahan A., Hoffman A., Eur. J. Pharm. Biopharm., 67, 96-105 (2007).

31) Sha X., Yan G., Wu Y., Li J., Fang X., Eur. J. Pharm. Sci., 24, 477486 (2005). 